\title{
JOHN DEWEY, SUA INFLUÊNCIA NA PEDAGOGIA ARGENTINA
}

\author{
Antonio Angel Taliercio ${ }^{1}$ \\ (1) https://orcid.org/0000-0002-5417-7050 \\ Margarita Sgró ${ }^{2}$ \\ (D) https://orcid.org/0000-0002-6346-5572
}

Resumo: John Dewey foi e é amplamente lido nos ambientes pedagógicos progressistas na Argentina. A produção de seu trabalho abrange um período da história da educação argentina cuja marca é fundamental. A estrutura política da Argentina, numa linha temporal, começa desde a Geração de 1880, a saber, Mitre, Avellaneda e o inovador Sarmiento até Perón, chegando aos dias atuais. Certamente a representação mais fiel da pedagogia da experiência e da nova escola é realizada pelas irmãs Cossettini na Escola Serena. O constante amor-ódio marca a relação entre Dewey e as políticas educacionais na Argentina.

Palavras-chave: Políticas educacionais. Nova escola. Pedagogia.

1 Professor Mestre em Educação, atua na disciplina de Filosofia especialmente ligada à formação de professores e gestão na área educacional. E-mail: aataliercio@gmail.com

2 Doutora em Educação pela Universidade de Campinas (UNICAMP) e exerceatividades de docência e pesquisa em sua universidade na Argentina, como ainda, a de coordenação noPrograma de Pósgraduação em Educação na mesma instituição (UNCPA). 


\section{JOHN DEWEY, HIS INFLUENCE IN ARGENTINE PEDAGOGY}

Abstract: John Dewey's production covers a period of the history of the Argentinian education, whose imprint is foundational. Argentina's political structure, in a line time, starts its history since generation of 1880, Mitre and Avellaneda, the innovation from Sarmiento up to Peron, reaching today. Certainly, the Cossenttini sisters at "The Serena Institution" carry out the most faithful representation of his pedagogy of the experience and the new school. The constant love-hate marks the relationship between Dewey and the educational policies in Argentina.

Keywords: Educational policies. New school. Pedagogy.

\section{JOHN DEWEY, SU INFLUENCIA EN LA PEDAGOGÍA ARGENTINA}

Resumen: John Dewey fue y es muy leído en los ambientes pedagógicos progresistas argentinos. La producción de su obra abarca un período de la historia de la educación argentina cuya impronta es fundacional. Desde el andamiaje político la línea histórica va desde la Generación de 1880, a saber, Mitre, Avellaneda y el innovador Sarmiento hasta Perón, llegando a la actualidad. Ciertamente la más fiel representación de la pedagogía de la experiencia y de la escuela nueva la llevan a cabo las hermanas Cossettini en La escuela Serena. El constante amor-odio marca la relación entre Dewey y las políticas educativas en Argentina.

Palabras clave: Políticas educativas. Escuela nueva. Pedagogía.

\section{Introdução}

Este trabajo se centrara en la lectura que tuvo la obra de John Dewey en los círculos de pedagogos de Argentina, el nivel de aceptación y los rechazos que sufrio sobre todo en los ambientes políticos contemporáneos a su obra.

Los objetivos seran los de visibilizar el recorrido historico y tambien pedagogico de la obra de Dewey en Argentina, el fuerte impacto en algunos maestros y pedagogos como lo veran en el caso de las hermanas Cossettini. Para este fin, recurrire a funtes historicas de autores argentinos y a la base de datos del portal $A B C$ del Ministerio de Educacion de Argentina. 
El camino a desandar esencialmente es el siguiente: ¿La aceptacion o rechazo de la obra de John Dewey, fue pedagógica opolítica?

Espero que sea un aporte para el lector.

\section{Contexto histórico, filosófico y pedagógico de la Pedagogía Progresista en Argentina.}

La obra de Dewey fue ampliamente leída en numerosos países, y esta lectura combinaba el avance de un movimiento pedagógico internacional con la construcción de verdaderos escenarios educativos nacionales en aquellos años. Esta combinación de pedagogías que circulaban internacionalmente y los campos de producción e interpretación nacionales parece haber sido particularmente importante en Argentina donde, desde su independencia del imperio español, la búsqueda de legitimación y solidez en el proceso de modernización había sido procesada frecuentemente con la ayuda de modelos y autoridades extranjeras, provenientes mayoritariamente de Europa y en menor medida de Estados Unidos.

Las elites argentinas imitaron con entusiasmo el modelo francés de escolarización centrado en una escuela primaria común, obligatoria, gratuita y de laicidad restringida e importaron conscientemente el positivismo francés, el cual se convirtió en la filosofía educativa fundante del sistema moderno escolar del país.

En la primera década del siglo XX, las fuerzas educativas que sostenían el programa positivista se impusieron definitivamente e imprimieron su particular diseño del sistema escolar, el cual contenía un programa pedagógico marcado por el cientificismo y por las propagaciones de vínculos pedagógicos racionalizadas y más bien autoritarias. Este programa asoció la expansión de la escolarización con la construcción del Estado-nación. Fue en este contexto en el cual el desafío representado por la obra de Dewey fue registrado y discutido por primera vez.

En general, la educación norteamericana constituía una referencia más bien marginal para los reformadores educativos argentinos, los cuales usualmente eran atraídos por las reformas llevadas a cabo en Europa. 
En el campo cultural, durante las primeras décadas del siglo XX, las referencias para la legitimación de la experiencia nacional eran buscadas tanto en la herencia hispánica o en figuras nativas como el gaucho o los indios.

Pero en el campo educativo, la preferencia por el modelo norteamericano era algo arcaica y se encontraba indisolublemente asociada a la personalidad por demás destacada de Domingo Faustino Sarmiento (1811-88), educador de fuste y presidente del país en el siglo XIX.

Sarmiento había viajado a los Estados Unidos en 1845 y 1846 por encargo del gobierno chileno, donde él estaba exiliado, y fue más tarde embajador de Argentina en aquél país. Renunció a este cargo para retornar a Buenos Aires como presidente electo en 1868. Era amigo de Horace y Mary Mann y estaba particularmente impresionado por la experiencia de desarrollo de un sistema escolar estructurado de fuerte base social, tal como se estaba dando en los estados federados norteamericanos en aquellos años. Cuando fue designado Director de Escuelas de la Provincia de Buenos Aires, ensayó reformas significativas a nivel local en las cuales dio impulso a los concejos escolares de distrito, copiados del modelo escolar de las escuelas comunes, como elemento central de estructuración del sistema escolar. Como presidente, promovió la inmigración de 65 graduadas de las escuelas normales norteamericanas, las cuales tuvieron una influencia significativa en la creación de las diversas escuelas normales en Argentina.

Pero la posición pro-norteamericana de Sarmiento sería un ejemplo solitario por varias décadas. Junto con una serie de causas políticas y económicas, entre ellas, la competencia entre Argentina y Estados Unidos por un rol líder en América Latina, el clima intelectual de la Argentina de fin de siglo experimentó la popularidad de un movimiento claramente anti-norteamericano conocido como arielismo, nombre que toma del libro de José Rodo, Ariel. Este autor, adepto a la tradición greco-latina, se vio Influenciado por la derrota española en la Guerra de Cuba de 1898 a manos de los Estados Unidos. El autor uruguayo se dirigió a la juventud latinoamericana denunciando los peligros de la expansión imperialista norteamericana en América Latina, la cual ya se percibía en la tentación materialista que estaría atravesando a la cultura latinoamericana. Este movimiento favoreció claramente respuestas antiliberales y debilitó el apoyo a alternativas educativas basadas en modelos vinculados a la experiencia norteamericana. 
Algunos investigadores han afirmado que Argentina, después de España, habría sido el país más receptivo a las ideas de Dewey en las primeras décadas del siglo XX. Sin embargo, esta afirmación carece de fuerte evidencia empírica para su fundamentación.

La primera traducción de Dewey al castellano del libro Mi credo pedagógico (original de 1897), fue publicada por el educador chileno Darío Salas en Santiago de Chile en 1908. Otras traducciones al castellano de libros de Dewey, por ejemplo, de La escuela y la sociedad (original de 1899) y Psicología del pensamiento (original de 1910), fueron publicadas respectivamente en Madrid (1915) y en Boston (1917). Los educadores españoles fueron particularmente activos en la traducción y publicación de la obra de Dewey hasta el desenlace de la Guerra Civil Española.

Solamente en el caso de Psicología del pensamiento puede apreciarse un interés argentino en la traducción y difusión de las ideas deweyanas. Ernesto Nelson (18731959), un inspector de escuelas que había concurrido a cursos de la Universidad de Columbia en Nueva York entre 1902 y 1906, escribió un prólogo para este libro que había sido traducido por el estudiante argentino Alejandro Jascalevich. Nelson y Jascalevich se habían conocido en la escuela secundaria experimental que dependía de la Universidad de La Plata, la primera universidad de investigación del país. Nelson era el director del Consejo escolar de esta institución reformista mientras que Jascalevich fue uno de sus primeros graduados. A pesar del hecho de que los textos publicados por Dewey durante las primeras tres décadas del siglo XX estaban vinculados a la educación, Nelson mostró un conocimiento profundo de su trabajo filosófico. Ernesto Nelson apuntó al hecho de que en la filosofía de Dewey democracia significaba siempre evolución y que el método científico constituían las marcas fundamentales de la civilización moderna (NELSON, 1917).

El nuevo estado de la vida colectiva habría descartado posiciones dogmáticas y sus fundamentos filosóficos tales como el concepto realista de verdad. Nelson argumentaba que democracia estaba asociada a la pérdida de las fuentes absolutas de autoridad y, consecuentemente, incitaba a los individuos a ser activos participantes de la vida social ya que todas las jerarquías entre razón y experiencia habían perdido su relevancia. Él reconocía el hecho de que el sistema filosófico de Dewey, aunque fuera una fuente de 
inspiración fundamental para reformas educativas, era más que una filosofía educativa y citaba al propio Dewey que había expresado que la filosofía no era otra cosa que una teoría educativa generalizada (NELSON, 1917).

Para Nelson, el tiempo en el cual educación y democracia tenían que convertirse en términos correlativos había llegado definitivamente a América Latina. Nelson convocó a una renovación general de las discusiones educativas las cuales debían basarse crecientemente en las lecciones de los hechos. La tarea urgente para todo el continente era la liberación del dogmatismo, de la convención y verdades a medias. Nelson era un norteamericanista convencido: fue unos de los fundadores del Instituto de Cultural Argentino Norteamericano (ICANA) y del Rotary Club, escribió numerosos libros y artículos sobre la cultura y las instituciones de aquél país. Por todo ello, fue acusado cariñosamente por los pedagogos del establishment positivista de cultivar un yanquismo enardecido.

Por supuesto, Nelson no fue el único educador argentino que tuvo un conocimiento extenso de la obra de Dewey. Raúl Díaz (1862-1918), uno de los inspectores en jefe del Ministerio de Educación de la Nación, también compartía muchas de las ideas de Dewey como parte de una política cultural democrática. Díaz había sido comisionado por el gobierno argentino a visitar los Estados Unidos en 1907-1908. Volvió profundamente impresionado por lo que había visto y trajo consigo una multitud de documentos sobre experiencias de gobierno escolar con participación de los niños. Enfatizó el compromiso de Dewey con la democracia y su consideración de la educación tanto desde el aspecto social como desde el psicológico.

Estos elementos de filosofía pragmatista y la introducción del tema de la democracia tanto en el currículum escolar como en la vida escolar distinguían a Nelson y a Díaz de otros autores más preocupados por el problema de la reforma de la enseñanza y de una nueva didáctica. Nelson, en particular, formuló su énfasis centrado en la democracia en un momento muy especial de la vida del país.

La política democrática alcanzó su primer momento de fuerte gravitación con la nueva ley electoral de 1912 y la consecuente victoria de la Unión Cívica Radical, un movimiento de las clases medias reformistas, en las elecciones de 1916. En ese momento, 
parecía posible y apropiado reorientar el sistema educativo bajo el nuevo gobierno radical que permaneció en el poder hasta $1930 .^{3}$

Pero, al mismo tiempo, estos gobiernos reformistas tenían que lidiar con una trayectoria heredada bastante exitosa de desarrollo escolar.

En el sistema educativo, los radicales dieron impulso a algunas reformas en las universidades, donde un movimiento estudiantil de base produjo considerables cambios a partir de 1918 y particularmente en la formación para el trabajo, pero no tocó la estructura educativa heredada. De esta manera, las políticas educativas favorecieron claramente la expansión de las estructuras existentes y casi no afectaron la cultura institucional de las escuelas argentinas.

Aunque la promesa de democracia política era central en la agenda social, la ola democrática no llegó al campo educativo en toda su fuerza. Sin embargo, las políticas educativas permitieron la existencia de numerosas iniciativas a nivel pedagógico-didáctico y esto determinó los intereses de los nuevos lectores de la obra de Dewey. En la atmósfera política y cultural particularmente liberal de la década de 1920, los educadores liberales y radicales que había enfrentado al status quo educativo y político comenzaron a traducir y leer textos de Dewey.

Aunque su figura era bien conocida entre los docentes, las lecturas de los maestros de la obra de Dewey que enfatizaban la cuestión de la democracia no sobrepasó los límites restringidos de ciertos círculos vanguardistas.

En los años siguientes, los educadores argentinos de diversas tendencias como el catolicismo, el marxismo o los espiritualistas liberales miraron a las ideas de Dewey con cierto desprecio y afirmaron que las mismas, por diversos motivos, no eran convenientes ni realizables para el desarrollo ulterior de las escuelas argentinas. Aunque Dewey fue respetado como educador y fue casi desconocido como filósofo se convirtió en un clásico moderno del pensamiento educativo, lo que condujo, irónicamente, a la marginalización de su obra y posiciones en las discusiones educativas de la época.

\footnotetext{
${ }^{3}$ El Golpe de Estado de Argentina de 1930 ocurrido el 6 de septiembre de 1930 en el cual un grupo de militares encabezado por el general José Félix Uriburu se apoderó del gobierno, derrocando al presidente radical Hipólito Yrigoyen y estableciendo una dictadura militar.
} 


\subsection{El Rechazo}

Este cambio ocurrió durante la década de 1930, cuando el movimiento escolanovista se convirtió paulatinamente, a partir de las lecturas que los pedagogos argentinos realizaron de la obra de Dewey, en la pedagogía oficial, incluso, del corto gobierno militar surgido del golpe de estado de 1930, todos con una orientación cultural ligada al catolicismo conservador.

Cuando Juan Bautista Terán (1880-1938), presidente del Consejo Nacional de Educación entre 1930 y 1932 y bajo el gobierno militar de José E. Uriburu, lideró un movimiento de espiritualización de la escuela de fuertes reminiscencias nacionalcatólicas, comenzaron las críticas y rechazos a ambas corrientes existentes, el positivismo y el pragmatismo, a las cuales acusaba de reducir al niño a un haz de instintos y tendencias. En su opinión, la escuela no debía ser un simple gimnasio para despertar y dar plenitud a las espontaneidades infantiles, como lo pretendían Dewey y Montessori. El objetivo de la educación, en la visión de Terán, debía ser la formación de un ser moral con libertad y responsabilidad. Terán consideró a Dewey como un filósofo naturalista, un simple heredero de Rousseau. El practicismo de Dewey deja de lado el fin puramente intelectual y el fin ético, o los considera implicados en la enseñanza adaptada a las conveniencias e imposiciones del medio en que ha de desenvolverse el niño. Es una aplicación estricta del pragmatismo, de la doctrina característica de su raza y de su país, según la cual la utilidade el fin supremo de la filosofía (TERÁN, 1932). Terán no sólo afirmaba que este sistema filosófico era éticamente peligroso, sino que lo condenaba como un fracaso histórico en el marco de la crisis de la economía mundial que había comenzado con la crisis estadounidense de 1929. En su mirada, la incapacidad de lograr bienestar material y su desdén de la cultura contemplativa y especulativa, la cultura "pura," habría llevado a ese país a la bancarrota. Obviamente, pensaba que no era conveniente seguir ese modelo sino, por el contrario, era necesario combatirlo. Terán defendió el espiritualismo como una filosofía educativa que implicaba el retorno a la inteligencia y que era opuesta al culto a la vida que sería típico del pragmatismo. Era un defensor del currículum humanista tradicional y negaba el valor educativo de la formación profesional porque esto significaba condenar al pueblo a vivir en el empirismo y cerrarles el acceso a las más altas posibilidades de la inteligencia. En su argumentación, el 
activismo tenía que estar subordinado a la disciplina, al orden y al respeto a las reglas, todos principios pedagógicos del gobierno militar.

La escuela nueva que Terán y sus colaboradores tenían en mente era similar a aquella que se desarrolló en la Italia fascista. La posición de Terán determinó durante la década de 1930 un clima donde las experiencias más radicalizadas al interior del escolanovismo argentino fueron perseguidas y muchos de sus adherentes fueron exonerados.

Este tipo de lectura de la obra de Dewey, aunque parezca tan limitado y tendencioso, no estaba confinada al catolicismo conservador de las derechas políticas. Puede sorprender el hecho de que se encuentren numerosas reminiscencias entre la visión de Dewey promovida por Terán y la de Aníbal Ponce, un profesor expulsado del Instituto del Profesorado por su afiliación al Partido Comunista. Ponce (1898-1938) condenó la pedagogía de Dewey como una expresión meramente utilitaria y puramente metodológica de la civilización burguesa norteamericana. Inscribió su posición crítica en un marxismo de fuerte carácter determinista. Veía la obra de Dewey en el marco de una tendencia metodológica de la escuela nueva que buscaba aumentar la performance de los alumnos ajustando la pedagogía a la personalidad del niño, tanto en su aspecto biológico como en el psicológico. El reclamo de Dewey por trabajos en grupo en la escuela era interpretado como una respuesta al fordismo capitalista que requería una nueva escuela basada en la socialización puerocéntrica de los alumnos en lugar del individualismo de las escuelas tradicionales. En la visión de Ponce, Dewey y Montessori representaban en modo los equivalentes de racionalización capitalista de la enseñanza.

La condena de Ponce sobre la obra de Dewey y sobre la totalidad del movimiento escolanovista estaba vinculada a un reduccionismo de clase que estructuraba su discurso y que lo llevó a negar las dimensiones nacionales específicas de la discusión educativa o cualquier otro tipo de mediación o matiz. Esta parcialidad lo llevó a aceptar las equivalencias discursivas establecidas en el campo educativo argentino que asociaban a la pedagogía de Dewey con un movimiento eficientista basado en la educación manual y en la formación por el trabajo (CARUSO; DUSSEL, 2009, p. 23). 
Otro aspecto importante de su rechazo de la obra de Dewey fue la admiración que tradicionalmente los partidos políticos de izquierda en Argentina habían desarrollado hacia la figura de Sarmiento y su misión civilizadora, haciendo que la izquierda formara parte de lo que podría denominarse la gramática pedagógica oficial. De esta manera, la izquierda criticó todas las reformas que habían intentado disputar la legitimidad del bachillerato clásico tradicional para la escuela secundaria.

Atacado tanto por pedagogos de la izquierda como de la derecha, Dewey tuvo pocos fieles seguidores en estos años. Entre ellos, se encuentra otro hombre de izquierdas que estuvo abocado a la propagación del ideario escolanovista con gran firmeza. El maestro Jesualdo Sosa (1905-1982), un uruguayo con vasta trayectoria y experiencia en Argentina, tenía una visión bastante diferente de la escuela nueva y de la obra de Dewey que la que se presentaba en la obra de Ponce. Consideraba que Dewey era uno de los partidarios burgueses más progresistas en el campo del trabajo escolar y que como figura intelectual estaría preparando la escuela socialista del futuro. Para Sosa, la propuesta de Dewey articulaba el trabajo escolar a la inteligencia y a la democracia. Jesualdo registraba también la influencia del fordismo y del taylorismo en la obra de Dewey, pero caracterizaba sus conceptos como evidentemente progresistas en comparación con los medios y objetivos educativos de sus predecesores. Uno de los comentarios negativos que Jesualdo hacía de la obra de Dewey era el papel jugado por la religión, una posición que en América del Sur estaba muy asociada al conservadurismo católico. Una vez más, Jesualdo se destacaba en el campo de las izquierdas políticas cuando reconocía que el término religión podría incluir también algún tipo de misticismo constructivo y necesario para la perfección humana y, por consiguiente, no condenaba la religión. Por ello, Jesualdo fue uno de los pocos pedagogos de la izquierda política que tendieron puentes al espiritualismo religioso imperante en algunos círculos intelectuales.

En el campo educativo mismo, la reacción de Terán fue aquélla que tuvo mayor impacto en las lecturas preponderantes de la obra de Dewey en las décadas de 1930 y 1940. Sin embargo, existe cierta evidencia de que algunos maestros estudiaban la obra de Dewey en círculos autónomos y alejados del control oficial. Correlativamente con estas lecturas críticas de la derecha y de la izquierda, la popularidad de Dewey entre los 
maestros creció cuando sus textos fueron más pedagógicos y menos políticos, pudiendo ser desvinculado, de esta manera, del credo liberal-democrático.

Esta transformación parece ser particularmente consistente con el perfil cada vez más técnico y despolitizado del movimiento escolanovista argentino, cuando sus voces más radicalizadas fueron censuradas y perseguidas.

\section{Dewey en Argentina - Contexto político.}

En argentina, el nombre de John Dewey fue una referencia persistente en los debates educativos de comienzos del siglo XX cuando su obra comenzó a ser leída y conocida por un círculo cada vez más grande de educadores. Como en el caso de otros países, Dewey fue considerado como un representante del liberalismo educativo y como un defensor del principio de actividad, de la pedagogía puerocéntrica ${ }^{4}$ y de la democracia educativa.

Asociado con este espectro de ideas, y especialmente identificado con una particular cultura, la norteamericana, que fue su condición de emergencia, la figura de John Dewey fue tan apreciada como denostada en los ambientes pedagógicos argentinos.

En este contexto de amor-odio podemos hacer referencia a una de las situaciones más llamativas e inesperadas donde su nombre fue evocado para la polarización de propuestas educativas. Fue una concentración política en un clima enrarecido preelectoral unos días antes de las elecciones generales de 1946 que dieron la victoria a Juan Domingo Perón. Durante este acto, que tuvo lugar en el conocido estadio Luna Park de la ciudad de Buenos Aires, el director del Instituto Nacional de Formación del Profesorado, Jordán Bruno Genta, un conocido ideólogo fascista, proclamo frente a 25000 maestros que habían acudido al evento bajo amenaza de despido: "La influencia perniciosa de John Dewey [...] debe ser erradicada de las escuelas argentinas [...]. La escuela nueva debe ser reemplazada por la escuela tradicional." (CARUSO; DUSSEL, 2009, p. 25) Este tipo de incidentes, que tuvieron lugar en un contexto represivo marcado por una dictadura militar de clara ideología nacional-católica, solidificó la mala reputación del naciente peronismo

\footnotetext{
${ }^{4}$ Sistema pedagógico cuya base esta en las necesidades inmediatas sentidas por el niño, este educando se presenta en constante cambio y evolución.
} 
en aquellos años. En todo caso, el mismo John Dewey comentó lacónicamente esta anécdota en una carta de lectores del semanario Time que había publicado el reportaje con las declaraciones de Genta: "El artículo sobre Argentina fue algo nuevo para mí. Bajo las circunstancias de ese país, lo tomo como un cumplido." (CARUSO; DUSSEL, 2009, p. 26). Es realmente revelador que el nombre Dewey haya jugado un papel tan central en el retrato que Genta pintaba a los enemigos de la tradición y del nacionalismo.

En estos meses dramáticos que siguieron el fin de la Segunda Guerra Mundial, el debate político se había polarizado al extremo. Uno de los polos era el de los partidos tradicionales que enfrentaban la candidatura de Perón y que iban desde los partidos más conservadores y tradicionales dominados por los grandes propietarios terratenientes de las provincias del norte pasando por los liberales progresistas de la Unión Cívica Radical y culminando por los socialistas y comunistas (una coalición denominada Unión Democrática). Estas fuerzas plantearon la confrontación política del momento como una simple oposición entre democracia y fascismo. El otro polo, el Partido Laborista que apoyaba la candidatura de Perón, construyó un claro antagonismo discursivo entre Perón y Braden (el embajador norteamericano que intervenía muy activamente en la política interior del país), una metáfora que evocaba la oposición entre el nacionalismo y el imperialismo norteamericano.

En este contexto, en el cual posiciones pro-norteamericanas y anti-norteamericanas estructuraban el antagonismo político del momento, la mención a John Dewey no era una mera referencia académica, sino que era utilizada para producir una serie de sentidos asociados.

\subsection{Dewey en Argentina - Contexto Pedagógico. La escuela de las Hermanas Olga y Leticia Cossettini.}

Una de las experiencias pedagógicas que se manifestaron entorno a John Dewey la encabezaron las hermanas Leticia y Olga Cossettini ${ }^{5}$ entre los años 1935 a 1950 en

\footnotetext{
${ }^{5}$ Las hermanas maestras santafesinas transformaron los formatos ortodoxos de la educación potenciando la estimulación y la creatividad de los niños. Fue pionera de que los estudiantes tengan un contacto con la naturaleza e impulsó las famosas excursiones a parques y plazas. Su hermana, Leticia Cossettini, fue clave en la aplicación de esa práctica educativa y aportó el arte (coro, teatro, títeres, danza, poesía y música) a la nueva Escuela que pensó Olga. Cossettini nació el 18 de agosto de 1898 en San Jorge, provincia de Santa Fe. Se recibió de maestra en Coronda. En 1930, asumió la Regencia de la Escuela Normal "Domingo de 
Rosario. Cuando la señorita Olga llega a la escuela Dr. Carrasco y la nombran directora, su primer objetivo fue que los maestros de la escuela entendieran sus ideas sobre la educación y fueran sensibles a estos conceptos que Olga Cossettini intentaba aplicar en su experiencia en la escuela.

Este proceso fue lento pero enriquecedor. En el testimonio de una ex alumna: "De una disciplina rígida, dirigida, pasamos a una autodisciplina, nacida del interior del niño hacia afuera" ${ }^{6}$.

En palabras de su hermana Leticia: "Los planes eran los del estado, pero vivificados por una experiencia con la vida circundante, con la gente, donde barrio, escuela y gente convergían"7.

La escuela incluía variadas experiencias creativas, había conciertos, donde cada chico llevaba su silla y se sentaba ansioso a esperar que empiece la música. Desde que el niño comenzaba a escuela se le daban acuarelas y a partir de la mancha elemental los dibujos iban adquiriendo un sentido.

Con el tiempo los medios expresivos de los chicos se iban enriqueciendo. Las observaciones sobre la flora, la fauna, los suelos, los mapas e incluso los personajes del barrio eran dibujados de diferentes maneras ya que respondían a una forma distinta de mirar, según cada individualidad de cada chico.

No había una clase de plástica o dibujo, sino que el arte estaba presente en el vivir cotidiano, porque la sensibilidad estaba en el vivir cotidiano. En las clases de teatro los chicos inventaban juegos rítmicos y representaban obras imprescindibles como La zapatera prodigiosa, de García Lorca.

Existía una relación solidaria de la escuela con su entorno, con el pueblo, con la gente. La escuela organizaba misiones culturales en donde los chicos llevaban al barrio actividades que interesaran a sectores de la sociedad que no podrían acceder a estas de otra forma. Los exalumnos tienen grabadas en su memoria las incontables experiencias

Oro" de Rafaela. Allí estuvo hasta 1935, donde consolidó sus principios pedagógicos. Olga murió a los 98 años, en 1997, en la misma vivienda del barrio Alberdi que actualmente es un museo y un centro cultural (BUENOS AIRES, 2019).

${ }^{6}$ Testimonio de una estudiante (LA ESCELA..., [2010]).

${ }^{7}$ Testimonio de Leticia Cossettini, hermana de Olga (LA ESCELA..., [2010]). 
educativas que les generó la escuela, dentro de las cuales estaban las visitas ilustres de artistas. En una ocasión se invita a Gabriela Mistral para que los chicos la conozcan, en otra a Juan Ramón Gimenez (autor de Platero y yo), en otra a Javier Villafañe un famoso titiritero, en su honor los chicos representaron Platero y yo en el teatro de títeres que armaron en la escuela.

Otra de las innovaciones fue el centro estudiantil cooperativo de la Escuela Carrasco. Muchas de las experiencias revolucionarias que tenía esta escuela a diferencia de los modelos estándares de educación tradicional, surgían de forma espontánea, por el propio interés de alumnos y docentes.

Cuando Leticia está dando las primeras clases en la escuela que dirige su hermana se encuentra con un grupo de chicos bastante inquieto que requiere gran cantidad de estímulos. Leticia da clases afuera, en el pasto, tomando sol o bajo los árboles. En una ocasión en que los chicos le piden un cuento ella relata uno clásico que recuerda, donde la protagonista debe hilar una gran cantidad de lino para terminar con su maleficio y unos pájaros que hablan hilan todo el lino para rescatarla. Al terminar el relato la señorita pregunta si alguno sabe imitar el canto de los pájaros a lo que algunos intrépidos responden que sí. De esa forma tan espontánea surge la creación del coro de pájaros en la escuela, donde había chicos que imitaban palomas, calandrias, horneros y gorriones entre otros.

La experiencia de la llamada escuela serena termina por decreto: Olga Cossettini es destituida de su cargo de directora, las razones son políticas. Los chicos sufren muchísimo la pérdida de Olga e incluso los maestros hacen una huelga, ya no quieren dar clases sin ella. El alejamiento de Olga de la escuela es violentamente radical, las autoridades tapian con maderas la puerta que conducían de la casa de Olga hacia la escuela, que quedaba en el piso de abajo. Resulta muy triste el final abrupto pero los testimonios de las personas que pasaron por esa escuela dejan claro que la experiencia pedagógica que les proporcionaron las hermanas Cossettini fue única, y les dio una base que los preparó para todas las experiencias posteriores en su vida.

Transcurrieron más de ocho décadas, y el modelo de escuela ideado por la docente rosarina Olga Cossettini conserva su valor original y más. Su propuesta de considerar a la educación desde un sentido amplio e innovador, priorizando la experiencia y la 
solidaridad, consiguió conformar uno de los proyectos de enseñanza que marcaron a la educación argentina del siglo XX.

Quebrar la barrera del enciclopedismo que proponía la escuela tradicional y pensar que los niños podían aprender saberes más allá de los métodos clásicos, fomentando las libertades y la imaginación, fue uno de sus objetivos como docente que dejó plasmado para la inmortalidad en su libro La escuela viva, de 1945.

\subsection{Dewey en Argentina - Escuela Normal mixta de Mercedes}

Otra experiencia educativa pionera del movimiento de la Escuela Nueva en Argentina fue llevada a cabo en la última década del siglo XIX, en la Escuela Normal Mixta de Mercedes, en la provincia de Buenos Aires, iniciada en el año 1887 y puesta en obra en 1890. El Director de ese establecimiento fue el pedagogo Carlos Vergara, quien implementó lo que ha sido considerado un anticipo pedagógico del escolanovismo.

El proyecto de Vergara propiciaba la autodeterminación de los alumnos en lo referente a la conducta y en su formación disciplinar, que daba prioridad a las tareas prácticas, a través de sus propias motivaciones, sin la imposición de castigos u otras formas de autoritarismo por parte de los maestros.

La experiencia de Vergara fue rechazada por sectores que ocupaban cargos políticos jerárquicos dentro del modelo educacional vigente y tal rechazo se canalizó a través del periódico de la comunidad de Mercedes, que llevó a cabo una campaña de desprestigio contra el Director y el equipo de maestros colaboradores. El análisis histórico posterior de este episodio condujo a algunos investigadores a sostener que tal medida reglamentaria extrema, por parte de las autoridades, respondió al carácter hegemónico que presentaba el recientemente creado Sistema Educativo Nacional, que respondía a la política educacional de la época.

\section{Testimonios. Olga por educadoras}

Como ilustración, pongo abajo algunos informes sobre el importante trabajo de esta educadora que "[...] transformó la educación tradicional de la primera mitad del siglo 
XX en la Argentina", según el sitio del Gobierno de la Provincia de Buenos Aires de 13 de marzo de 2019 (BUENOS AIRES, 2019).

3.1 MARIANA DAMATO - maestra de las primarias 28 y 50 de la ciudad de Necochea:

Por estos días quiero hacer referencia a Olga, una colega a la que siento cerca y con la que comparto su legado: ese espíritu de innovar, de probar experiencias nuevas, de hacer de la escuela una escuela nueva, viva. Una escuela que siga latiendo a pesar de todo, donde se trabaje con entusiasmo, con pasión, a la par de los compañeros y con el sólo objetivo de brindarles a los alumnos una escuela viva. Así como Olga pensó en transformar la educación, siento que todas las mujeres, cada una desde su rol debemos apostar también, arriesgarnos, sabiendo que siempre lo que hacemos con pasión dará buenos resultados (BUENOS AIRES, 2019).

3.2 VERÓNICA BUSTOS - maestra de la Primaria 26 de Saladillo:

Olga y Leticia supieron innovar, rompiendo los paradigmas impuestos de la época, y apostaron a romper las barreras de las desigualdades generando la articulación entre la estética y la pedagogía. El niño artista era el eje; la posibilidad expresiva, el motor. La experiencia derrumbó bloques horarios, cuadrículas por materia, los márgenes y los renglones, el límite entre la escuela y la comunidad. En la propuesta pedagógica que ellas realizaron hay una continuidad, entre el arte y la ciencia. Se ve en los cuadernos porque era el modo en que se planteaban las actividades. Así, en los paseos que realizaban por el barrio, la observación era una clara actividad de enseñanza, se recogían algunos materiales y se dibujaba. Todo tenía una articulación. La ciencia estaba presente con continuidad porque ellas planteaban una integración de los lenguajes en los trabajos cotidianos (BUENOS AIRES, 2019).

3.3 SANDRA LESTARPE - directora de la Primaria 62 de Saladillo:

Olga y Leticia Cossettini mujeres educadoras de honda vocación y espíritu sensible. Vanguardistas, desafiaron los límites de su tiempo y propusieron a los niños que transitaron sus aulas, la experiencia vital del aprendizaje. El cuerpo se volvió instrumento de conocimiento y la multiplicidad de sensaciones les posibilitó sentir, saber y conocer con la intensidad de un genuino aprender.

Hoy, volvemos a esta genuina experiencia educadora, para sentir que es posible que la escuela se vuelva espacio en donde la niñez construya un proyecto de vida sensible y superador. Así lo testimonian cada uno de los que fueron partícipes de la Escuela de la señorita Olga (BUENOS AIRES, 2019). 
3.4 - MARÍA MÓNICA GENIZ - ex directora de la Escuela Padre Respuela de Junín:

Muchas de las experiencias pedagógicas que aplicaba Olga están presentes en las aulas de hoy y siguen sosteniendo los mismos principios de aquellas aulas: respeto por la personalidad infantil, eliminación de las fronteras entre la escuela y la comunidad, la educación como un hecho social, rechazo de toda forma de discriminación, igualdad en la consideración a los niños de diversa procedencia y a los colegas, entre otros. Pienso en lo difícil que son los cambios de paradigma y me pregunto cómo habrá reaccionado la comunidad frente a las innovaciones promovidas por Olga y su hermana Leticia en el marco de las teorías de la Nueva Escuela o Escuela Activa. Pero nada obstaculiza cuando hay saber, convicción y firmeza en la toma de decisiones (BUENOS AIRES, 2019).

\section{Conclusión}

Luego de las lecturas de los trabajos de investigadores que siguieron las huellas de Dewey en la pedagogía argentina queda claro que los educadores argentinos recurrieron repetidas veces a la obra de John Dewey en el marco de una búsqueda de modelos de reforma educativa. Sin embargo, su reputación indiscutida como uno de los mayores intelectuales del campo educativo contrastó con una visión particularmente selectiva de su obra y con una recepción muy débil de la misma en términos institucionales. De hecho, partes sustantivas de sus investigaciones fueron marginalizadas en una lectura parcial que produjo una versión de Dewey como educador despolitizado.

Las múltiples vinculaciones entre democracia, educación y escolarización sugeridas por Dewey fueron reducidas a una didáctica, dejando de lado la profundidad de su pensamiento filosófico, de parte de un movimiento que reflejó las trayectorias políticas e intelectuales de los adeptos al movimiento de la escuela nueva en el país.

En ese tiempo histórico las tradiciones liberal y católica de lectura de modelos y referencias extranjeras fueron puestas en un campo de discusión junto con las luchas que organizaron los juegos de enfrentamiento en el campo político del de ese período, y particularmente con el clima de polarización política del momento, es el caso de los primeros gobiernos elegidos democráticamente y de las dictaduras militares. 
Estos fueron algunos de los elementos que marcaron fuertemente la recepción de Dewey en la Argentina. La política partidaria forjo un ostracismo, esto sumado a los miedos de la caída de verdades impuestas, han sido los responsables de que la obra de Dewey no tuviese mayor difusión.

\section{Referências}

BUENOS AIRES. Dirección General de Cultura y Educación. Olga Cossettini, una maestra que cambió la escuela. Buenos Aires: ABC, 13 mar. 2019. Disponible en: http://www.abc.gov.ar/olga-cossettini-una-maestra-que-cambio-la-escuela Acesso en: 4 abr. 2020.

CARUSO, M.; DUSSEL, I. Dewey en Argentina (1916-1946): tradición, intención y situación en la producción de una lectura selectiva. Encuentros sobre Educación, Mendonza, v. 10, p. 23-41, 2009.

LA ESCUELA de la Señorita Olga. [S. I.: s. n., 2010]. 1 vídeo (46min 54). Publicado pelo canal J. S. Disponible en: https://vimeo.com/17048589 Acesso en: 4 abr. 2020.

NELSON, E. Prologo: introducción a la edición española. En: DEWEY, J. Psicología del pensamiento. Boston: D.C. Health and Co., 1917. Disponible en: https://www.unav.es/gep/IntroduccionPsicologiaPensamiento.html

TERÁN, J. B. Prólogo: espiritualizar nuestra escuela: la instrucción primaria argentina en 1931. Buenos Aires: Librería del Colegio, 1932. 